\title{
Review Article: Instrumental Analysis of Olopatadine Hydrochloride, Oxeladine Citrate, Amlodipine Besylate and Xipamide
}

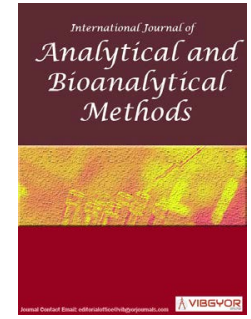

\section{Mahmoud M Sebaiy ${ }^{1 *}$, Hisham E Abdellatef ${ }^{2}$, Magda M Elhenawee ${ }^{2}$, Mohamed A Elmosallamy $^{3}$ and Mustafa Kh Alshuwaili ${ }^{3,4}$}

${ }^{1}$ Department of Medicinal Chemistry, Faculty of Pharmacy, Zagazig University, Egypt

${ }^{2}$ Department of Analytical Chemistry, Faculty of Pharmacy, Zagazig University, Egypt

${ }^{3}$ Department of Chemistry, Faculty of Science, Zagazig University, Egypt

${ }^{4}$ Ministry of Education, Iraq

\begin{abstract}
In this literature review, we will introduce all reported methods that have been developed for determination of the antihistaminic drug, olopatadine $\mathrm{HCl}$, the cough suppressant drug oxeladine citrate, and certain antihypertensive drugs such as amlodipine besylate and xipamide in their pure form, combined form with other drugs, combined form with degradation products, and in biological samples. We also will shed the light on the most important combination of drugs that are used for treatment of allergy and hypertension.
\end{abstract}

\section{Olopatadine Hydrochloride}

Olopatadine hydrochloride 11-[(Z)-3-(Dimethylamino) propylidene]-6,11-dihydrodibenzo [b,e]oxepin-2-acetic acid hydrochloride (Figure 1) [1].

\section{Physical properties}

Olopatadine Hydrochloride is the hydrochloride salt form of olopatadine. Olopatadine hydrochloride is white crystalline powder. It is freely soluble in water [1].

\section{Pharmacological action}

Olopatadine hydrochloride is used to treat allergic conjunctivitis (itching eyes), as it inhibits the release of histamine from mast cells. It is a relatively

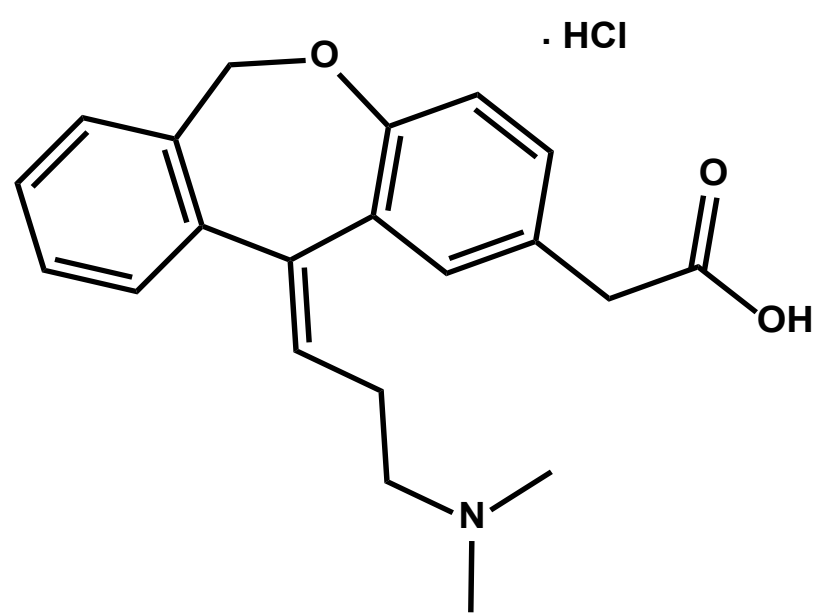

Figure 1: $\mathrm{C}_{21} \mathrm{H}_{23} \mathrm{NO}_{3} . \mathrm{HCl}$ (M.wt. 373.87). selective histamine $\mathrm{H} 1$ antagonist that inhibits the

*Corresponding author: Mahmoud M Sebaiy, Department of Medicinal Chemistry, Faculty of Pharmacy, Zagazig University, Zagazig, Egypt, Tel: 01062780060 , Fax: 0552303266

Accepted: June 11, 2020; Published: June 13, 2020

Copyright: () 2020 Sebaiy MM, et al. This is an open-access article distributed under the terms of the Creative Commons Attribution License, which permits unrestricted use, distribution, and reproduction in any medium, provided the original author and source are credited.

Sebaiy et al. Int J Analyt Bioanalyt Methods 2020, 2:010 
in vivo and in vitro type 1 immediate hypersensitivity reaction including inhibition of histamine induced effects on human conjunctival epithelial cells [2].

\section{Methods of determination}

Spectroscopic methods: A novel simple, accurate, sensitive and economical spectrophotometric method has been established and validated for the determination of the antihistaminic drug, olopatadine hydrochloride in both pure form and eye drops [3]. The spectrophotometric method is divided into four procedures ( $A, B, C \& D)$. The method is based on the oxidation of the studied drug by a known excess of $\mathrm{KMnO}_{4}$, followed by measuring the decrease in absorption $(\triangle \mathrm{A})$ of $\mathrm{KMnO}_{4}$ in $2 \mathrm{M} \mathrm{H}_{2} \mathrm{SO}_{4}$ (A), in $0.1 \mathrm{M} \mathrm{NaOH}(\mathrm{B})$ both at $504 \mathrm{~nm}$ or measuring the increase in absorption of added methyl orange in $0.1 \mathrm{M} \mathrm{NaOH}$ (C) at $443 \mathrm{~nm}$ or measuring the change of $\mathrm{KMnO}_{4}$ into green colour in $1 \mathrm{M} \mathrm{NaOH}$ (D) at $605 \mathrm{~nm}$. The detection limit is reported to be $1.05,0.62,0.40$ and $0.60 \mu \mathrm{g} / \mathrm{ml}$ for procedures $A$, $B, C$ and $D$, respectively showing a high degree of sensitivity. The proposed method was successfully validated according to FDA guidelines for the determination of the drug in eye drops with a highly precise recovery and very low relative standard deviation. Finally, the method was compared statistically with a reference method showing equal accuracy, reproducibility and no significant difference with the reported one.

Spectrophotometric determination of olopatadine hydrochloride in eye drops and tablets has been reported by [4]. In this studying, the validated three UV spectrophotometric methods have been proposed for the determination of olopatadine hydrochloride in different dosage forms (eye drops and tablets) and pure drug. Linear regression equation (LRE), standard absorptivity (SA) and first order derivative (FOD) method were validated for linearity, accuracy, precision and robustness according to ICH guideline to assure reliability of the developed methods.

Simultaneous determination of ketorolac tromethamine and olopatadine hydrochloride by UItraviolet Spectrophotometry (Simultaneous equation method) is described by [5]. The method involves determination of ketorolac tromethamine at $320.0 \mathrm{~nm}$ and olopatadine hydrochloride at 206.0 $\mathrm{nm}$. The \% assay of the drugs was found to 98.99 \pm 0.7619 and $99 \% \pm 1.3228$ for ketorolac trometh- amine and olopatadine hydrochloride respectively. Validation of the proposed method was carried out for its linearity, accuracy, precision, LOD, and LOQ, according to ICH guidelines. The recovery of the ketorolac tromethamine and olopatadine hydrochloride were found to $100.18 \pm 0.072167 \%$ and $99.55 \pm$ $0.010516 \%$ for ketorolac tromethamine and olopatadine hydrochloride, respectively.

Application of alizarin red $\mathrm{S}$ as an ion-pair reagent has been used for the spectrophotometric determination of olopatadine hydrochloride in pharmaceutical formulation [6]. This method was based on the formation of (yellow) ion - association complex with alizarin red $\mathrm{S}$ as chromogenic reagent in acidic medium, which is extracted into chloroform. The complex has a maximum absorbance at $425 \mathrm{~nm}$. All variables affecting the reaction were studied and optimized. The method was successfully applied to the determination of olopatadine hydrochloride in its pharmaceutical formulation.

UV spectrophotometric method was developed for the estimation of olopatadine hydrochloride from its ophthalmic dosage form by zero order (Do) derivative spectroscopic method [7]. The correlation coefficient of olopatadine hydrochloride is 0.999 and drug recovery is $99.95 \%$. The developed UV spectrophotometric method was validated according to ICH guidelines with respect to accuracy, precision, ruggedness, specificity, LOD $(0.213 \mu \mathrm{g} /$ $\mathrm{ml}$ ) and LOQ $(2.133 \mu \mathrm{g} / \mathrm{ml})$.

First order derivative spectroscopic method has been developed for the estimation of olopatadine hydrochloride and ambroxol hydrochloride in their mixture synthetic [8]. The wavelength maxima for olopatadine hydrochloride and ambroxol hydrochloride are $299.20 \mathrm{~nm}$ and $307.40 \mathrm{~nm}$, respectively. For the first order derivative spectroscopic method, the Zero crossing points (ZCPs) for olopatadine hydrochloride and ambroxol hydrochloride were obtained at $299.80 \mathrm{~nm}$ and $307.20 \mathrm{~nm}$ respectively in the distilled water using Shimadzu 1800 UV-visible double beam spectrophotometer.

UV spectrophotometric estimation of olopatadine hydrochloride in bulk and pharmaceutical dosage form by area under curve and second order derivative methods was developed [9]. In this method the standard and sample solutions of olopatadine hydrochloride were prepared in methanol. Olopatadine hydrochloride was estimated at $224 \mathrm{~nm}$ for the second order derivative UV - spectrophotomet- 
ric method $(A)$, while in area under curve (AUC) method (B) the zero order spectrum of olopatadine hydrochloride was measured in between $220 \mathrm{~nm}$ to $230 \mathrm{~nm}$. These methods were tested and validated for various parameters according to $\mathrm{ICH}$ guidelines. The precision expressed as relative standard deviation were of $0.1311 \%$ and $0.5889 \%$ for the above two methods.

Chromatographic methods: Chromatographic analysis was carried out for olopatadine in hydrophilic interaction liquid chromatography [10]. In this paper, Box-Behnken design was utilized, and on the basis of the obtained models factor effects were examined.

A simple RP-HPLC method was developed and validated for rapid assay of olopatadine in pharmaceutical dosage form and wavelength was set at $246 \mathrm{~nm}$ [11].

A rapid and sensitive liquid chromatography tandem mass spectrometry method was performed for determination of olopatadine concentration in human plasma [12]. The sample preparations were carried out by protein precipitation with the addition of acetonitrile followed by liquid-liquid extraction with ethyl acetate/dichloromethane after internal standard (IS, amitriptyline) spiking.

Stability indicating high-performance column liquid chromatography and high-performance thin-layer chromatography methods for the determination of olopatadine hydrochloride in tablet dosage form was performed [13]. This paper describes two simple methods for estimation of olopatadine hydrochloride (OLO) in tablet dosage form. The first method is a stability-indicating isocratic RP-HPLC method, precoated silica gel aluminum sheets were used as the stationary phase; the mobile phase was methanol-chloroform-ammonia.

Application of high performance liquid chromatographic technique for olopatadine hydrochloride and its impurity in ophthalmic solution was carried out [14]. The present work describes the ion pair RP-HPLC method for determination of olopatadine hydrochloride and its related substances. The separation of olopatadine hydrochloride and impurities were achieved at $220 \mathrm{~nm}$.

Capillary electrophoresis method: A validated capillary electrophoretic method for the determination of olopatadine and its application to a pharmaceutical preparation of eye drops was per-

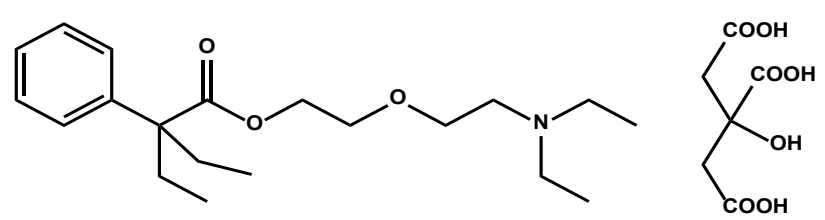

Figure 2: $\mathrm{C}_{26} \mathrm{H}_{41} \mathrm{NO}_{10}$ (M.wt. 527.611).

formed [15]. The validated capillary zone electrophoretic method for the determination of olopatadine hydrochloride is described at pH 9.5, with 25 $\mathrm{kV}$.

Miscellaneous methods: Voltammetric determination of olopatadine hydrochloride in Bulk drug form and Pharmaceutical Formulations was carried out [16]. The voltammetric behavior of olopatadine was investigated at hanging mercury drop electrode using cyclic voltammetry and differential pulse voltammetry.

\section{Oxeladine Citrate}

Oxeladine Citrate, 2-[2-(diethylamino)ethoxy] ethyl 2-ethyl-2-phenylbutanoate; 2-hydroxypropane-1,2,3-tricarboxylic acid (Figure 2) [17].

\section{Physical properties}

Oxeladine citrate is white or almost white, crystalline powder. It is very soluble in water [17].

\section{Pharmacological action}

Oxeladine is a cough suppressant. It is a highly potent and effective drug used to treat all types of cough of various etiologies such as irrigative cough, allergic cough, psychogenic cough, cough in patients with heart disease, tracheitis, bronchitis and other conditions [18].

\section{Methods of determination}

Spectroscopic methods: A spectrophotometric method for the assay of some drugs including the oxeladine citrate that containing quaternary ammonium or tertiary amine moieties is described. The method is based on the interaction of these drugs with quercetin after its oxidation with $\mathrm{N}$-bromo succinimide at 528-560 nm [19].

Chromatographic methods: Determination of oxeladine citrate in the presence of two of its degradation products by HPTLC and HPLC was performed [20]. Objectives of this study are hydrolytic and the oxidative degradation of oxeladin citrate, to prepare its alkaline induced hydrolytic degradation product, $\alpha, \alpha$-diethyl benzeneacetic acid and its 
$\mathrm{N}$-oxide degradation product, diphenylmethoxy- $\mathrm{N}$, $\mathrm{N}$-dimethyl ethanamine $\mathrm{N}$-oxide and to develop and validate HPTLC-densitometric and RP-HPLC methods for determination of oxeladin citrate.

High performance liquid chromatographic determination of oxeladin citrate and oxybutynin hydrochloride and their degradation products was carried out [21]. Two high performance liquid chromatographic (HPLC) methods are presented for the determination of $\mathrm{OL}$ and $\mathrm{OB}$. The first method was based on HPLC separation of OL using a mobile phase consisting of acetonitrile and phosphoric acid. The second method was based on HPLC separation of $\mathrm{OB}$ using a mobile phase consisting of acetonitrile and potassium dihydrogen phosphate/ diethyl amine. Quantitation was achieved with UV detection at $220 \mathrm{~nm}$.

Determination of oxeladin in human plasma by gas chromatography-mass spectrometry was described [22]. A capillary gas chromatographic method with mass-selective detection was developed for the determination of oxeladine in human plasma. Plasma samples were alkalinized and extracted using hexane:isoamyl alcohol.

Determination of oxeladine in human sera by gas-liquid chromatography with thermionic detection was carried out [23]. This paper describes a gas-liquid chromatographic (GLC) method developed for the determination of oxeladine in serum samples in bioavailability tests of oxeladine from tablet and linctus formulations.

Miscellaneous methods: Two novel potentiometric sensors were developed for the quantification of olopatadine hydrochloride and oxeladine citrate in pure forms, pharmaceutical preparations and human plasma [24]. The both sensors were fabricated on incorporating the olopatadine and oxeladine tetraphenylborate ion-pairs as electroactive materials in plasticized poly (vinyl chloride) matrix membranes with o-nitrophenyl octyl ether. They displayed Nernstian and near Nernstian responses over the concentration ranges of $1.0 \times 10^{-5}$ - $1.0 \times 10^{-2} \mathrm{~mol} \mathrm{~L}^{-1}$ oxeladine and olopatadine with cationic slopes of 60 and $50 \mathrm{mV}$ decade-1, respectively. Detection limits were reported to be $4.4 \times$ $10^{-6}$ and $5.0 \times 10^{-6} \mathrm{~mol} \mathrm{~L}^{-1}$ while the response times were 3.0 and 18 seconds for oxeladine and olopatadine, respectively. The useful ranges of $\mathrm{pH}$ were 2.5-8.5 and 3.0-4.5 for oxeladine and olopatadine, respectively. The life extents were long ( 3 months)

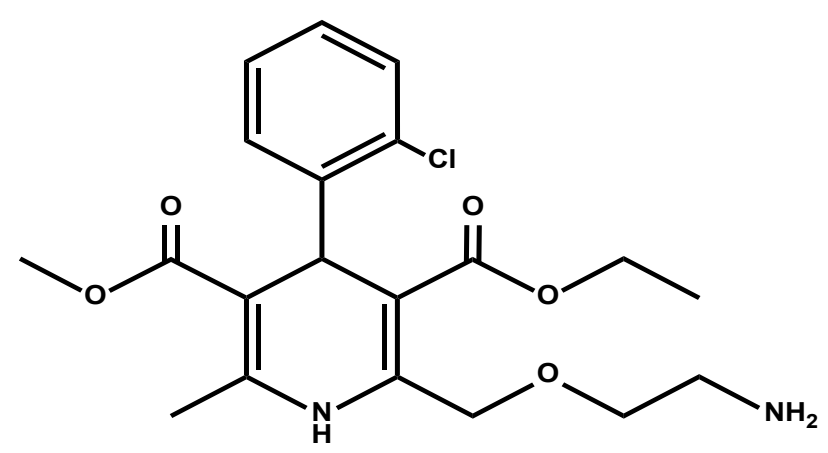

Figure 3: $\mathrm{C}_{26} \mathrm{H}_{31} \mathrm{ClN}_{2} \mathrm{O}_{8} \mathrm{~S}$ (M.wt. 567.05).

for both sensors. The sensors were successfully used for the assay of oxeladine and olopatadine in pharmaceutical dosage forms with a recovery of $100.0 \%$ for both drugs. Also the oxeladine sensor was used for the estimation of the drug in human plasma with an average recovery of $99.49 \%$.

Plastic membrane, carbon paste and multiwalled carbon nanotube composite coated copper wire sensors for determination of oxeladin citrate using batch and flow injection techniques was described [25]. The proposed sensors in this work are three novel a PVC plastic membrane sensors, a carbon paste sensor and a multiwalled carbon nanotube (MWCNT) composite coated copper wire sensor. The sensors are based on the oxeladin-phosphotungstate ion associate as electroactive material and dibutyl phthalate as solvent mediator.

\section{Amlodipine Besylate}

Amlodipine Besylate, is 3-ethyl 5-methyl 2-((2-aminoethoxy)methyl)-4-(2-chlorophenyl)-6-methyl-1,4-dihydropyridine-3,5-dicarboxylate benzenesulfonate (Figure 3) [17].

\section{Physical properties}

Amlodipine besylate is a white crystalline powder. It is slightly soluble in water, and sparingly soluble in ethanol [17].

\section{Pharmacological action}

Amlodipine Besylate is the besylate salt of amlodipine, a synthetic dihydropyridine with antihypertensive and antianginal effects. Amlodipine inhibits the influx of extracellular calcium ions into myocardial and peripheral vascular smooth muscle cells, thereby preventing vascular and myocardial contraction. This results in a dilatation of the main coronary and systemic arteries, decreased myocardial contractility, increased blood flow and oxygen 
delivery to the myocardial tissue, and decreased total peripheral resistance [18].

\section{Methods of the determination}

Spectroscopic methods: A Spectrophotometric determination of amlodipine besylate $(A M B)$ in pharmaceutical preparations using gresol red (GR) reagent was performed [26]. The proposed method is based on the formation of colored chloroform extractable ion-pair equal-molecular complexes of amlodipine with gresol red in britton $\mathrm{pH} 2$ buffer. The extracted complexes showed absorbance maxima at $420 \mathrm{~nm}$.

Spectrophotometric method of estimation of amlodipine besylate using hydrotropic solubilization was carried out [27]. The spectrophotometric method was based on using $2 \mathrm{M}$ urea solution as hydrotropic solubilizing agent for the estimation of poorly water-soluble drug amlodipine besylate. Various organic solvents such as methanol, chloroform, dimethyl formamide and acetonitrile have been employed for solubilization of poorly water-soluble drugs to carry out spectrophotometric analysis at $243 \mathrm{~nm}$.

Native and synchronous spectrofluorimetric methods for simultaneous determination of amlodipine besylate/valsartan combination in tablets were used [28]. This study is based on two methods. The first method depends on measurement of native fluorescence intensity of both drugs at emission $460 \mathrm{~nm}$ and $385 \mathrm{~nm}$ using excitation $390 \mathrm{~nm}$ and $227 \mathrm{~nm}$ for AM and VS respectively in water. The second method utilizes synchronous fluorimetric quantitative screening of the emission spectra of AM and VS at 375 and $285 \mathrm{~nm}$, respectively using $\Delta \lambda$ of $80 \mathrm{~nm}$.

Sequential spectrophotometric method for the simultaneous determination of amlodipine, valsartan, and hydrochlorothiazide in coformulated tablets was described [29]. In this method three techniques were used, namely, direct spectrophotometry, ratio subtraction, and isoabsorptive point. Amlodipine (AML) was first determined by direct spectrophotometry and then ratio subtraction was applied to remove the AML spectrum from the mixture spectrum. Hydrochlorothiazide (HCT) could then be determined directly without interference from valsartan (VAL) which could be determined using the isoabsorptive point theory.

Simultaneous spectrophotometric estimation of amlodipine besylate and telmisartan in tablet dosage form was described [30]. Two spectrophotometric methods for the determination of amlodipine besylate (AMB) and telmisartan (TEL) by simultaneous equation method and first order derivative method in combined dosage form. The simultaneous equation method is based on measurement of absorbance at $367 \mathrm{~nm}$ and $292 \mathrm{~nm}$ as two wavelengths selected for quantification of $A M B$ and TEL. The second method is first order derivative based on the measurement of absorbance at $270 \mathrm{~nm}$ and $295 \mathrm{~nm}$ as two wavelength selected as for quantification of $A M B$ and TEL.

Two spectrophotometric methods for the determination of amlodipine besylate [31]. These methods are based on the formation of an ion association complex between the drug and an acidic dye, bromothymol blue, which is extractable into chloroform (Method-A), or by the formation of an oxidative coupling product with 3-methyl-2-benzothiazolinone hydrazone hydrochloride (MBTH) in the presence of ceric ammonium sulphate (Method-B).

One direct titrimetric method and two indirect spectrophotometric methods for the determination of amlodipine besylate (ADB) are described [32]. The methods uses bromate-bromide mixture and two-dyes, namely Methyl Orange and Indigo Carmine, as reagents. In titrimetry (Method A), an acidified solution of ADB is titrated directly with bromate-bromide mixture using methyl orange as an indicator. Spectrophotometry involves the addition of known excess of bromate-bromide mixture to an acidified solution of ADB and the determination of the residual bromine based on its ability to bleach the dyes Methyl Orange (Method B) or Indigo Carmine (Method C) quantitatively.

Spectrofluorimetric determination of amlodipine in human plasma without derivatization was carried out [33]. The type of solvent, the wavelength range, and the range of $A D$ concentration were selected to optimize the experimental conditions. The method developed in this study can be directly and easily applied for the determination of amlodipine in the plasma without derivatization in plasma.

Spectrophotometric determination of amlodipine besylate using 2-hydroxy naphthaldehyde as a derivatizing reagent was performed [34]. Spectrophotometric method for the determination of the amlodipine besylate by derivatization with 2 hy- 
droxy naphthaldehyde. The molar absorptivity of amlodipine besylate after derivatization was calculated at $416 \mathrm{~nm}$.

Chromatographic methods: An HPLC method had been developed and validated for rapid simultaneous separation and determination of the two antihypertensive drugs, amlodipine and xipamide in human plasma within 5 minutes [35]. Drugs are extracted from plasma using methanol, the environmentally benign solvent, for protein precipitation technique. Separation was carried out on a Thermo Scientific ${ }^{\circ}$ BDS Hypersil $C_{8}$ column $(5 \mu \mathrm{m}, 2.50 \times 4.60$ $\mathrm{mm}$ ) using a mobile phase of methanol: $0.025 \mathrm{M}$ $\mathrm{KH}_{2} \mathrm{PO}_{4}(70: 30, v / v)$ adjusted to $\mathrm{pH} 3.49$ with ortho-phosphoric acid at ambient temperature. The flow rate was $1 \mathrm{ml} / \mathrm{min}$ and maximum absorption was measured using DAD detector at $235 \mathrm{~nm}$. The retention times of amlodipine and xipamide were recorded to be 4.51 and 3.38 minutes, respectively, indicating a shorter analysis time. Limits of detection were reported to be 0.17 and $0.25 \mu \mathrm{g} / \mathrm{ml}$ for amlodipine and xipamide, respectively, showing a high degree of the method sensitivity. The method was then validated according to FDA guidelines for the determination of the drugs clinically in human plasma specially regarding pharmacokinetic and bioequivalence studies.

A high-performance liquid chromatographic (HPLC) method for the determination of amlodipine drug in dosage form using 1,2-naphthoquine 4-sulfonate (NQS) was developed [36]. The method is based on the formation of a brown color product from the reaction between AML and NQS. The nucleophilic substitution reaction proceeds quantitatively at $\mathrm{pH} 13$ buffer solution with absorption maximum at $465 \mathrm{~nm}$, and a mobile phase of combination potassium dihydrogen orthophosphate in water:acetonitrile were used for separation and quantification.

Determination of amlodipine in human plasma by electrospray ionization LC-MS/MS method: Validation and its stability studies were carried out [37]. High-performance liquid chromatography combined with electrospray ionization (ESI) tandem mass spectrometry (LC-MS/MS) method, operating in the positive ionization mode, for quantifying of amlodipine in human plasma using tizanidine as internal standard, were extracted by simple one step liquid/liquid extraction with a mixture of diethyl ether/dichloromethane.
Liquid chromatography electrospray ionization-tandem mass spectrometry (LC-MS/MS) method for the quantification of amlodipine in human plasma, with gliclazide as an internal standard was used [38]. The analyte was extracted with ethyl acetate and analyzed on a diamond. The mobile phase was composed of methanol- ammonium acetate with gradient flow rates and gradient conditions.

High performance thin layer chromatography (HPTLC) method with densitometry for simultaneous determination of amlodipine and enalapril from pharmaceutical preparation was developed [39]. Separation was performed on aluminum-backed silica gel HPTLC plates as stationary phase and using a mobile phase comprising of Toluene: Isopropanol: Glacial acetic acid (GAA): Methanol and a mobile phase was used per chromatography run.

Simultaneous determination of amlodipine besylate and atorvastatin calcium in binary mixture by spectrofluorimetry and HPLC coupled with fluorescence detection was performed [40]. In the HPLC method, separation of AM and AT was achieved within 8 minutes using acetonitrile:phosphate buffer as the mobile phase.

HPLC method for the assay of amlodipine in human plasma was described [41]. The assay involves derivatization with 4-chloro-7-nitrobenzofurazan (NBD-Cl), solid-phase extraction on a silica column and isocratic reversed-phase chromatography with fluorescence detection. Nortriptyline hydrochloride was used as an internal standard.

A Validated HPLC method for determination of amlodipine in human plasma and its application to pharmacokinetic studies was used [42]. The method involves simple, one-step extraction procedure. The separation was performed on Nucleosil column, the wavelength was set at $239 \mathrm{~nm}$.

Quantitative determination of amlodipine besylate, losartan potassium, valsartan and atorvastatin calcium by HPLC in their pharmaceutical formulations was described [43]. The method was performed by reversed phase high performance liquid chromatography using a mobile phase ammonium acetate buffer ( $\mathrm{pH} 5.5)$ : acetonitrile with detection at $240 \mathrm{~nm}$.

Capillary electrophoresis methods: Simultaneous determination of amlodipine and telmisartan from pharmaceutical products by way of capillary electrophoresis was developed [44]. Electrophoret- 
ic parameters such as buffer concentration and $\mathrm{pH}$, system temperature, applied voltage, and others were studied. The separation was achieved, while the order of migration was amlodipine followed by telmisartan.

Miscellaneous methods: Voltammetric and square-wave anodic stripping determination of amlodipine besylate on gold electrode was performed [45]. The oxidative behaviour of amlodipine besylate was studied. The gold electrode and Au/o-MWCNT (oxidized multi-wall carbon nanotubes) were used for determination of amlodipine besylate standard and as a content of alopres tablet, in $0.05 \mathrm{M} \mathrm{NaHCO}_{3}$ and in phosphate buffer $(\mathrm{pH}=11)$ by cyclic voltammetry and square-wave anodic stripping voltammetry. The gold electrode is better catalyst for anodic oxidation of amlodipine besylate than glassy carbon.

Determination of amlodipine besylate by adsorptive square-wave anodic stripping voltammetry on glassy carbon electrode in tablets and biological fluids was described [46]. The adsorptive and electrochemical behavior of amlodipine besylate on a glassy carbon electrode was explored in britton-robinson buffer solution by using cyclic and square-wave voltammetry. Cyclic voltammetric studies indicated the oxidation of amlodipine besylate at the electrode surface through a single two-electron irreversible step and fundamentally controlled by adsorption. The solution conditions and instrumental parameters were optimized for the determination of the authentic drug by adsorptive square-wave stripping voltammetry.

Electro-oxidation and simultaneous determination of amlodipine and atorvastatin in commercial tablets using carbon nanotube modified electrode was developed [47]. Amlodipine besylate and atorvastatin calcium have been determined by the simultaneous voltammetric method at a multiwalled carbon nanotubes: Graphite (MWCNTs:G) paste electrode. In comparison with a glassy carbon electrode, the prepared electrode showed an increase in the peak current because of the high electroactive surface area and excellent electronic conductivity of MWCNTs.

Voltammetric and liquid chromatographic (LC) methods for the determination of amlodipine besylate (AML) and rosuvastatin calcium (ROS) were carried out for the first time [48]. Detailed electrochemical behavior and simultaneous vol- tammetric determination of $\mathrm{AML}$ and ROS were investigated in detail using glassy carbon electrode (GCE). High-performance liquid chromatography (HPLC) and ultra-performance liquid chromatography (UPLC) were also developed for the comparison. Voltammetric method exhibited linear dynamic responses for the simultaneous assay of AML and ROS.

Electroanalytical studies and simultaneous determination of amlodipine besylate and atorvastatin calcium in binary mixtures using first derivative of the ratio voltammetric methods was performed [49]. The electrochemical behavior of atorvastatin and amlodipine at a glassy carbon electrode has been studied using different voltammetric techniques. This technique depends on the measuring of first derivative of the ratio voltammograms of each concentration as a function of the increased concentrations. DP and SW voltammetric methods depend on first derivative of the ratio-voltammetry by measurements of the selected potentials for amlodipine and atorvastatin.

\section{Xipamide}

Xipamide is 4-chloro-N-(2,6-dimethylphenyl)-2-hydroxy-5-sulfamoylbenzamide (Figure 4) [17].

\section{Physical properties}

Xipamide is white or almost white, crystalline powder. It is practically insoluble in water, freely soluble in acetone, soluble in ethanol, soluble in chloroform, and soluble in diethyl ether [17].

\section{Pharmacological action}

Xipamide is a sulfonamide - based diuretic. It is used for treatment of hypertension and edema, including that associated with heart failure [18].

\section{Methods of the determination}

Spectroscopic methods: New and sensitive spectrophotometric method for determination

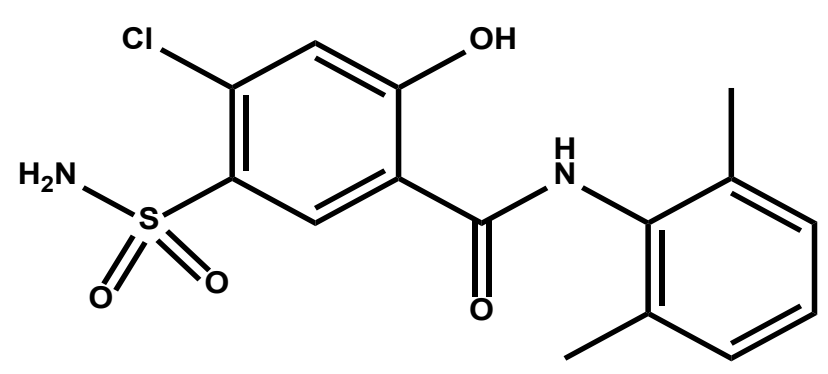

Figure 4: $\mathrm{C}_{15} \mathrm{H}_{15} \mathrm{CIN}_{2} \mathrm{O}_{4} \mathrm{~S}$ (M.wt. 354.805). 
of xipamide in pure and dosage forms by complexation with $\mathrm{Fe}(\mathrm{III}), \mathrm{Cu}(\mathrm{II}), \mathrm{La}(\mathrm{III}), \mathrm{UO}_{2}(\mathrm{II}), \mathrm{Th}(\mathrm{IV})$ and $\mathrm{ZrO}$ (II) ions was carried out [50]. The method is based on the reaction of xipamide with $\mathrm{Fe}(\mathrm{III})$, $\mathrm{Cu}(\mathrm{II}), \mathrm{La}(\mathrm{III}), \mathrm{UO}_{2}$ (II), Th(IV) and $\mathrm{ZrO}$ (II) ions to form stable colored complexes. Optimum conditions for determination, stoichiometry and stability of the formed complexes have been determined.

Spectrophotometric and spectrodensitometric determination of triamterene and xipamide in pure form and in pharmaceutical formulation was developed [51]. UV-spectrophotometric for the determination of triamterene (TRM) and xipamide (XIP) in their binary mixture, formulated for use as a diuretic, without previous separation, based on the four methods. Method $A$ is the isoabsorptive point spectrophotometry, method $B$ is the ratio subtraction spectrophotometry, method $C$ is a chemometric-assisted spectrophotometry and method $D$ is a TLC-densitometry.

Stability-indicating spectrofluorimetric methods for the determination of metolazone and xipamide in their tablets. Application to content uniformity testing was described [52]. The proposed method is based on the measurement of the native fluorescence of MET in methanol at $437 \mathrm{~nm}$ after excitation at $238 \mathrm{~nm}$ and XPM in alkaline methanolic solution at $400 \mathrm{~nm}$ after excitation at $255 \mathrm{~nm}$.

Chromatographic methods: High performance liquid chromatography for simultaneous determination of xipamide, triamterene and hydrochlorothiazide in bulk drug samples and dosage forms was performed [53]. Chromatographic separation was carried out in less than two minutes. The separation was performed stationary phase with an isocratic elution system consisting of ortho phosphoric acid ( $\mathrm{pH} 2.3$ ) and acetonitrile (ACN) as the mobile phase in the ratio of 50:50, at $2.0 \mathrm{~mL} \mathrm{~min}^{-1}$ flow rate at room temperature. Detection was performed at $220 \mathrm{~nm}$.

Stability-Indicating HPLC-DAD method development, validation, and stress degradation Studies for triamterene and xipamide in their combined tablet dosage form was studied [54]. Separation of the two drugs from all the degradant peaks was achieved within $11 \mathrm{~min}$ and mobile phase consisting of acetonitrile and phosphate buffer adjusted to $\mathrm{pH} 4$ delivered at a flow rate of $1 \mathrm{~mL} \mathrm{~min}^{-1}$ using gradient elution system. The drugs were quantified at $220 \mathrm{~nm}$ using photodiode array detector, based on peak area.
Determination of the non-thiazide diuretic xipamide in pharmaceuticals and urine by HPLC with amperometric detection was developed [55]. The mobile phase consisted of a mixture water:acetonitrile, $\mathrm{pH}$ 4.3. The compound is monitored at +1325 $\mathrm{mV}$ with an amperometric detector equipped with a glassy carbon working electrode. A liquid-liquid or solid-liquid extraction prior to chromatographic analysis was done to avoid the interferences found in urine matrix.

Bioavailability study of triamterene and xipamide using urinary pharmacokinetic data following single oral dose of each drug or their combination was described [56]. The HPLC separation was performed on a RP stainless-steel with a gradient elution system of phosphate buffer adjusted to $\mathrm{pH}$ 4.0 and methanol as the mobile phase. The method was used to determine the urinary excretion profile and to calculate different urinary pharmacokinetic parameters following oral dose of their combination compared with single oral doses of each drug and hence comparing their bioavailability.

High-performance liquid chromatographic determination of xipamide in human plasma was carried out [57]. It involves extraction of the drug and an internal Standard, into diethyl ether - isopropanol aporation of the organic phase and analysis of the reconstituted residue. The drug and internal standard are detected by ultraviolet absorption at $225 \mathrm{~nm}$.

Miscellaneous methods: Electrochemical characterization of xipamide using cyclic and square wave voltammetry was described [58]. The electrochemical behaviour of xipamide was studied at glassy carbon electrode in Britton-Robinson buffer solution of different $\mathrm{pH}$, using cyclic voltammetry and square wave voltammetry. Voltammetric results showed a reversible redox and diffusion-controlled process. The oxidation and reduction peaks were found at potentials of $0.375 \mathrm{~V}$ and $0.321 \mathrm{~V}$ at $\mathrm{pH}$ 5.0, respectively.

Photoinduced electron transfer photo degradation of photosensitive diuretic drug-xipamide was developed [59]. In the present study, the photochemical behavior of xipamide was investigated in the presence of both electron donor and acceptor. When xipamide (1) was irradiated with a high-pressure mercury lamp under anaerobic conditions in the presence of electron-donor N,N-dimethylaniline (DMA) it afforded photoproduct (2) and in the 
presence of an electron-acceptor 1,4-dicyanonaphthalene (DCN) it afforded photoproduct (3). The product formation was explained through the photo induced electron transfer mechanism. Products were isolated and identified on the basis of IR, NMR and mass spectral studies.

$\mathrm{PH}$ Assist for highly selective determination of xipamide by the enhancement of the green emission of $\mathrm{Tb}^{3+}$ optical sensor was performed [60]. The method depends on the enhancement of the green emission band of $\mathrm{Tb}^{3+}$ at $545 \mathrm{~nm}$ in the presence of different concentration of xipamide at $\mathrm{pH} 4.1$ and $\lambda_{\mathrm{ex}}=320 \mathrm{~nm}$ in acetonitrile. The photo physical properties of the green emissive $\mathrm{Tb}^{3+}$ complex have been elucidated.

\section{Conclusion}

This literature review represents an up to date survey about all reported methods that have been developed for determination of olopatadine $\mathrm{HCl}$, oxeladine citrate, amlodipine besylate and $\mathrm{xi}$ pamide in their pure form, combined form with other drugs, combined form with degradation products, and in biological samples such as liquid chromatography, spectrophotometry, spectroflourimetry, voltammetry, etc...

\section{References}

1. (2013) United States Pharmacopoeia. National Formulary, USA, 4566.

2. Ohmori K, Hasegawa K, Tamura T, Miyake K, Matsubara $M$, et al. (2004) Properties of olopatadine hydrochloride, a new antiallergic/antihistamine drug. Arzneimittelforschung 54: 809-829.

3. Sebaiy MM (2019) Colurimetric determination of olopatadine hydrochloride oxidation-reduction products in pure form and eye drops. Austin J Anal Pharm Chem 6: 1115.

4. Jain D, Basniwal PK (2013) Spectrophotometric determination of olopatadine hydrochloride in eye drops and tablets. J of Pharm Res 12: 48-52.

5. Thakkar Hiren S, Patel Pinkal R, Patel Rina B, Patel Bhavika B, Patel Shirish R, et al. (2013) Simultaneouse determination of olopatadine hydrochloride and ketorolac by ultraviolet spectrophotometric. Inventi Rapid-Pharm Anal \& Quality Assur 23: 113-119.

6. Elbashir AA, Abdalla FAA (2013) Application of alizarin red $S$ as an ion-pair reagent for the spectrophotometric determination of olopatadine hydrochloride in pharmaceutical formulation. America Academic \& Schol Res J 5: 22-27.
7. Zaheer Z, Khan FN, Shaikh DA, Khan S, Baig MS, et al. (2016) Zero order derivative spectroscopic estimation of olopatadine hydrochloride from eye drops. J Inn Pharm Biol Sci 3: 103-107.

8. Shukla MH, Patel AP, Patel MG, Patel DP, Shah RR (2015) Development and validation of first order derivative spectroscopic method for estimation of olopatadine hydrochloride and ambroxol hydrochloride order in their synthetic mixture. Pharma Science Monitor 6: 119-131.

9. Rele RV (2015) UV-spectrophotometric estimation of olopatadine hydrochloride in bulk and pharmaceutical dosage form by area under curve and second order derivative methods. RJPT 8: 265-269.

10.Maksić J, Jovanović M, Rakić T, Popović I, Ivanović D, et al. (2014) Chromatographic analysis of olopatadine in hydrophilic interaction liquid chromatography. J Chromatogr Sci 53: 680-686.

11. Raul SK, Kumar BR, Patnaik AK, Rao NN (2012) A RPHPLC method development and validation for the estimation of olopatadine in bulk and pharmaceutical dosage forms. Asian J Res Chem 5: 1395-1398.p

12.Zhu P, Wen YG, Fan XP, Zhou ZL, Fan RX, et al. (2011) $A$ rapid and sensitive liquid chromatography-tandem mass spectrometry method for determination of olopatadine concentration in human plasma. J Anal Toxicol 35: 113-118.

13.Varghese SJ, Kumar AM, Ravi TK (2011) Stability-indicating high-performance column liquid chromatography and high-performance thin-layer chromatography methods for the determination of olopatadine hydrochloride in tablet dosage form. J AOAC Int 94: 1815-1820.

14. Rele RV, Warkar CB (2011) Application of high performance liquid chromatographic technique for olopatadine hydrochloride and its impurity in ophthalmic solution. Int J Chem Sci 9: 601-614.

15.Güray T, Turan T, Tunçel M, Uysal UD (2017) A validated capillary electrophoretic method for the determination of olopatadine and its application to a pharmaceutical preparation of eye drops. J AOAC Int 100: 206-211.

16.Sreedhar NY, Sreenivasulu A, Kumar MS, Nagaraju M (2012) Voltametric determination of olopatadine hydrochloride in bulk drug form and pharmaceutical formulations. Int J Pharm Sci Res 3: 2517-2522.

17.(2017) British Pharmacopoeia. HM Stationery Office, London, 11: II-472.

18.Sweetman S, Martindale (2009) The complete drug reference. $\left(36^{\text {th }}\right.$ edn), London. 
19. Mohamed FA, Mohamed AMI, Mohamed HA, Hussein SA (1996) Utility of quercetin for determination of some tertiary amine and quaternary ammonium salts. Talanta 43: 1931-1939.

20.Ali N, Abdelkawy M, Abdelhamid NS (2013) Simultaneous determination of paracetamol and diphenhydramine hydrochloride mixture in the presence of their degradation products. IOSR J Pharm Biol Sci 6: 44-52.

21.El-Gindy A (2005) High performance liquid chromatographic determination of oxeladin citrate and oxybutynin hydrochloride and their degradation products. II Farmaco 60: 689-699.

22.Lartigue-Mattei C, Galmier MJ, Chabard JL, Beyssac E, Aiache JM (1995) Determination of oxeladin in human plasma by gas chromatography-mass spectrometry. Biomed Chromatogr 9: 32-35.

23.Salvesen B, Haugland T (1981) Determination of oxeladin in human sera by gas-liquid chromatography with thermionic detection. J of Chromatogr B: Biomed Sci and Appl 225: 463-468.

24.Sebaiy MM, Elmosallamy MA, Elhenawee MM, Alshuwaili MK (2019) Poly (Vinyl Chloride) matrix membrane sensors for the quantification of olopatadine and oxeladine in pharmaceutical preparations and human plasma. Microchem J 147: 170-175.

25.Zayed SI, Issa YM (2013) Plastic membrane, carbon paste and multiwalled carbon nanotube composite coated copper wire sensors for determination of oxeladin citrate using batch and flow injection techniques. J Bra Chem Soc 24: 585-594.

26.Badran R, Al-khateeb MJ (2015) A spectrophotometric determination of amlodipine besylate (amb) in pharmaceutical preparations using gresol red (gr) reagent. Inter J Chem Tech Res 8: 229-236.

27.Bernard S, Mathew M, Senthilkumar KL (2011) Spectrophotometric method of estimation of amlodipine besylate using hydrotropic solubilization. J Appl Pharma Sci 01: 177-180.

28. Mohammed TAEF (2015) Native and synchronus spectrofluorimetric methods for simultaneous determination of amlodipine besylate and valsartan combination in tablets. Asian J Sci Techno 6: 16901698.

29.Darwish HW, Hassan SA, Salem MY, El-Zeany BA (2013) Sequential spectrophotometric method for the simultaneous determination of amlodipine, valsartan, and hydrochlorothiazide in coformulated tablets. Inter J Spectro 2013: 1-8.

30.Pawar PY, Raskar MA, Kalure SU, Kulkarni RB (2012)
Simultaneous spectrophotometric estimation of amlodipine besylate and telmisartan in tablet dosage form. Der Pharma Chem 4: 725-730.

31.Sridhar K, Sastry CSP, Reddy MN, Sankar DG, Srinivas KR (1997) Spectrophotometric determination of amlodipine besylate in pure forms and tablets. Anal Lett 30: 121-133.

32. Basavaiah K, Chandrashekar U, Nagegowda P (2006) Titrimetric and modified spectrophotometric methods for the determination of amlodipine besylate using bromate-bromide mixture and two dyes. Sci Asia 32: 271-278.

33. Kadioglu Y, Ozturk M (2012) Spectrofluorimetric determination of amlodipine in human plasma without derivatization. Braz J Pharm Sci 48: 719-725.

34.Almani F, Memon AH, Mughal UR, Memon N, Maheshwari ML, et al. (2010) Spectrophotometric determination of amlodipine besylate using 2-Hydroxynaphthaldehyde as a derivatizing reagent. Asian J Chem 22: 1205-1213.

35.Sebaiy MM, Abdellatef HE, Elmosallamy MA, Alshuwaili MK (2020) Isocratic HPLC method for simultaneous determination of amlodipine and xipamide in human plasma. Open J Anal Bioanal Chem 4: 001006.

36.Osman RAM, Elbashir AA (2017) A high-performance liquid chromatographic (HPLC) method for the determination of amlodipine drug in dosage form using 1, 2-Naphthoquine-4-Sulfonate (NQS). J Anal Pharm Res 6: 2-7.

37.Sirikatitham A, Panrat K, Tanmanee N (2008) Determination of amlodipine in human plasma by electrospray ionization LC-MS/MS method: Validation and its stability studies. Songklanakarin Journal of Science \& Technology 30: 455-462.

38.Lv CM, Wei CM, Bu FL, Chen R, Wang XL, et al. (2013) Determination of amlodipine in human plasma by LC-MS/MS and its bioequivalence study in healthy Chinese subjects. Pharmaco \& Pharmacy 4: 191-196.

39.Tamboli AM, Khan NI, Bathe RS, Ansari AM (2014) HPTLC method for simultaneous determination of amlodipine besylate and enalapril maleate in pharmaceutical formulation. Int J Biomed Adv Res 5: 237241.

40.Moussa BA, El-Zaher AA, Mahrouse MA, Ahmed MS (2013) Simultaneous determination of amlodipine besylate and atorvastatin calcium in binary mixture by spectrofluorimetry and HPLC coupled with fluorescence detection. Anal Chem 8: 107-115.

41.Tatar S, Atmaca S (2001) Determination of amlodip- 
ine in human plasma by high-performance liquid chromatography with fluorescence detection. Journal of Chromatography B: Biomedical Sciences and Applications 758: 305-310.

42.Zarghi A, Foroutan SM, Shafaati A, Khoddam A (2005) Validated HPLC method for determination of amlodipine in human plasma and its application to pharmacokinetic studies. II Farmaco 60: 789-792.

43. Hafez HM, Elshanawane AA, Abdelaziz LM, Kamal MM (2014) Quantitative determination of amlodipine besylate, losartan potassium, valsartan and atorvastatin calcium by HPLC in their pharmaceutical formulations. Pharm Anal Acta 5: 2-9.

44.Modroiu A, Hancu G, Vlad RA, Stăcescu Ș, Soare R, et al. (2016) Simultaneous determination of amlodipine and telmisartan from pharmaceutical products by way of capillary electrophoresis. Current Issues in Pharmacy and Medical Sciences 29: 42-46.

45.Stoiljković ZZ, Avramov Ivić ML, Petrović SD, Mijin DZ, Stevanović SI, et al. (2012) Voltammetric and squarewave anodic stripping determination of amlodipine besylate on gold electrode. Int J Electrochem Sci 7: 2288-2303.

46.Gazy AAK (2004) Determination of amlodipine besylate by adsorptive square-wave anodic stripping voltammetry on glassy carbon electrode in tablets and biological fluids. Talanta 62: 575-582.

47. Mohammadi A, Moghaddam AB, Eilkhanizadeh K, Alikhani E, Mozaffari S, et al. (2013) Electro-oxidation and simultaneous determination of amlodipine and atorvastatin in commercial tablets using carbon nanotube modified electrode. Micro \& Nano Lett 8: 413-417.

48. Karadas-Bakirhan N, Gumustas M, Uslu B, Ozkan SA (2016) Simultaneous determination of amlodipine besylate and rosuvastatin calcium in binary mixtures by voltammetric and chromatographic techniques. lonics 22: 277-288.

49.Dogan-Topal B, Bozal B, Demircigil BT, Uslu B, Ozkan SA (2009) Electroanalytical studies and simultaneous determination of amlodipine besylate and atorvastatine calcium in binary mixtures using first derivative of the ratio-voltammetric methods. Electroanalysis 21: $2427-2439$.

50.Gaber M, Khedr AM, El-Kady AS (2011) New and sensitive spectrophotometric method for determination of xipamide in pure and dosage forms by complex- ation with Fe (III), CU (II), La (III), UO2 (II), Th (IV) and ZrO (II) ions. Int Res J Pharm Pharmacol 1: 215-220.

51.Wagieh NE, Abbas SS, Abdelkawy M, Abdelrahman MM (2010) Spectrophotometric and spectrodensitometric determination of triamterene and xipamide in pure form and in pharmaceutical formulation. Drug Testing and Analysis 2: 113-121.

52. Walash MI, El-Enany N, Eid MI, Fathy ME (2014) Stability-indicating spectrofluorimetric methods for the determination of metolazone and xipamide in their tablets. Application to content uniformity testing. J Fluor 24: 363-376.

53.Abd El-Hay SS, Hashem H, Gouda AA (2016) High performance liquid chromatography for simultaneous determination of xipamide, triamterene and hydrochlorothiazide in bulk drug samples and dosage forms. Acta Pharma 66: 109-118.

54.El-Kimary El (2016) Stability-indicating HPLC-DAD method development, validation, and stress degradation studies for triamterene and xipamide in their combined tablet dosage form. Acta Chromatogr 28: 79-98.

55.Legorburu MJ, Alonso RM, Jimenez RM (1999) Determination of the non-thiazide diuretic xipamide in pharmaceuticals and urine by HPLC with amperometric detection. J Liqu Chromatogr Rela Technol 22: 735-746.

56. Maher HM, Youssef RM, Eman I, Hassan EM, Barary MA (2012) Bioavailability study of triamterene and xipamide using urinary pharmacokinetic data following single oral dose of each drug or their combination. J Pharm Biomed Anal 61: 78-85.

57.Dadgar D, Kelly M (1988) High-performance liquid chromatographic determination of xipamide in human plasma. Analyst 113: 229-231.

58. Attia AK, Hendawy HM (2017) Electrochemical characterization of xipamide using cyclic and square wave voltammetry. Res Rev Electrochemistry 8: 104-111.

59.Gupta A, Iqbal J, Ahmad W, Zaheer MR (2014) Photoinduced electron transfer photodegradation of photosensitive diuretic drug-xipamide. J Taibah Univ Sci 8: 64-70.

60.Attia MS, Yuossef AO, Diab M, El-Shahat MF (2014) $\mathrm{pH}$ assist for highly selective determination of xipamide by the enhancement of the green emission of Tb3+ optical sensor. J Advances Chem 10: 27202727. 